\section{P3 EFFICACY OF A PHYSIOTHERAPY, SPEECH AND LANGUAGE THERAPY INTERVENTION (PSALTI) ON HEALTH RELATED QUALITY OF LIFE (HRQOL) FOR PATIENTS WITH REFRACTORY CHRONIC COUGH: A RANDOMISED CONTROL TRIAL}

${ }^{1}$ SAF Chamberlain, ${ }^{2}$ SS Birring, ${ }^{3} \mathrm{~L}$ Clarke, ${ }^{4} \mathrm{~A}$ Douiri, ${ }^{5} \mathrm{SM}$ Parker, ${ }^{6} \mathrm{SJ}$ Fowler, ${ }^{7} \mathrm{~J}$ Hull, ${ }^{7} \mathrm{KF}$ Chung, ${ }^{1} \mathrm{~A}$ Pandyan, ${ }^{8} \mathrm{R}$ Garrod. ${ }^{1}$ School of Health and Rehabilitation, Keele University, Keele, UK; ${ }^{2}$ Division of Asthma, Allergy and Lung Biology, King's College London, London, UK; ${ }^{3}$ Speech and Language Therapy Department, King's College Hospital, London, UK; ${ }^{4}$ NIHR Biomedical Centre and Department of Primary Care and Public Health Sciences, King's College London, London, UK; ${ }^{5}$ Respiratory Medicine, Northumbria Healthcare NHSFT, North Tyneside General Hospital, North Shields, UK; ${ }^{6}$ Respiratory and Allergy Research Group, University of Manchester, Manchester and Lancashire Teaching Hospitals NHS Foundation Trust, Preston, UK; ${ }^{7}$ NIHR Respiratory Biomedical Research Unit, Royal Brompton NHS Foundation Trust and Imperical College London, London, UK; ${ }^{8}$ Denmark Hill Campus, King's College London, London, UK

\subsection{6/thoraxjnl-2014-206260.153}

Introduction Refractory chronic cough has a significant negative impact on HRQoL. There are currently limited effective antitussive therapies. Few studies have explored the effectiveness of nonpharmacological interventions for refractory chronic cough. This study investigated the efficacy of PSALTI on HRQoL for people with refractory chronic cough in a multi-centred RCT.

Methods Participants were recruited across five NHS hospitals trusts. 76 participants were randomised to PSALTI or placebo (equal attention) intervention. PSALTI consisted of education, laryngeal hygiene and hydration advice, cough control techniques and psycho-educational counselling. Placebo consisted of general education on exercise, diet, stress and relaxation. Both groups attended 4 weekly sessions of 1:1 therapy. HRQoL was measured at baseline, four weeks (end of treatment) and 3 month follow up by Leicester cough questionnaire (LCQ). Cough reflex sensitivity was assessed at baseline and four weeks by capsaicin cough challenge (C2, C5, concentration that caused first urge to cough $(\mathrm{Cu})$ ) and was analysed by geometric means (GM). Outcomes between groups were analysed using ANCOVA.

Results The PSALTI $(\mathrm{n}=35)$ and Placebo groups $(\mathrm{n}=41)$ were well matched $(\mathrm{p}>0.05)$ for age [mean (SD)] 58(15) vs. $56(11)$ years; gender $71 \%$ vs $63 \%$ females; cough duration [median (IQR)] 60(30 to 126$)$ vs $48(24$ to 126$)$ months and baseline LCQ [mean (SD)] 10.4(3.6) vs 11.9(3.5). At four weeks HRQoL improved in both groups, mean LCQ increase in PSALTI was 3.4 (95\% CI 2.26 to 4.55$)$ vs placebo 1.7 (95\% CI 0.78 to 2.54); difference in LCQ change between groups was $1.5(95 \%$ CI 0.27 to $7.31, \mathrm{p}=0.02)$ points more on average in the PSALTI group. This effect is greater than the MCID for LCQ and was sustained at 3 months (mean difference change between groups after 4 weeks to 3 months was -0.28 (95\% CI -1.83 to 1.38 ). There was a significant increase in $\mathrm{Cu}$ in the PSALTI group compared to placebo (GM(SD) change $2 \mu \mathrm{m}$ (5.07) vs $0.612 \mu \mathrm{m}$ (3.26), p = 0.02). There was no significant difference in cough reflex sensitivity between the groups $(\mathrm{C} 2, \mathrm{p}=0.46 ; \mathrm{C} 5, \mathrm{p}=0.74)$.

Conclusions PSALTI significantly improved HRQoL compared with equal attention placebo intervention and this improvement was sustained at three months. PSALTI also significantly increased $\mathrm{Cu}$ compared to placebo.
P4

ESTABLISHING A ROLE FOR TRPV1 ON SENSORY NERVES IN COPD ASSOCIATED CHRONIC COUGH

${ }^{1}$ MA Wortley, 'SA Maher, ${ }^{1} \mathrm{SJ}$ Bonvini, , ED Dubuis, ${ }^{1} \mathrm{~J}$ Nasra, ${ }^{2} \mathrm{~K}$ Holt, ${ }^{2} \mathrm{R}$ Dockry, ${ }^{2} \mathrm{~S}$ Sen, ${ }^{2} \mathrm{D}$ Singh, ${ }^{2} \mathrm{JA}$ Smith, ${ }^{3} \mathrm{P}$ Round, ${ }^{3} \mathrm{~S}$ Gilbert, ${ }^{3} \mathrm{~V}$ Marchant, ${ }^{3} \mathrm{~J}$ Ford, ${ }^{1} \mathrm{MA}$ Birrell, ${ }^{1} \mathrm{MG}$ Belvisi. ${ }^{1}$ National Heart and Lung Institute, Imperial College, London, UK; ${ }^{2}$ University of Manchester, University Hospital of South Manchester, Manchester, UK; ${ }^{3}$ Ario Pharma Limited, Cambridge, UK

\subsection{6/thoraxjnl-2014-206260.154}

Background An increase in cough reflex sensitivity to capsaicin in COPD has been described in some studies, suggesting a role for TRPV1 in the disease phenotype. We utilised a guinea-pig cigarette-smoke (CS) exposure COPD model to investigate an enhanced cough phenotype, and evaluate the role of TRPV1 using a novel clinical-ready inhibitor, XEN-D0501. ${ }^{1}$ Furthermore, we confirmed enhanced cough responses in COPD patients using a dose-response capsaicin challenge to determine $\mathrm{E}_{\text {Max. }}$

Methods Guinea-pigs were exposed to air/cigarette smoke (CS) for $1 \mathrm{~h}$, twice daily, for 8 days. Coughs evoked by aerosolised capsaicin $(30 \mu \mathrm{M})$, depolarisation of isolated vagus nerve tissue induced by capsaicin $(1 \mu \mathrm{M})$, or increases in intracellular calcium $\left[\mathrm{Ca}^{2+}\right]_{\mathrm{i}}$ to capsaicin $(1 \mu \mathrm{M})$ in airway-terminating (i.n. DiI stained) jugular and nodose neurons were evaluated. Vagus nerve was obtained from human non-smoker/smoker subjects for similar assessment. Coughs evoked by capsaicin (4 inhalations, 0.49$1000 \mu \mathrm{M}$ ) were recorded in COPD and compared with healthy controls.

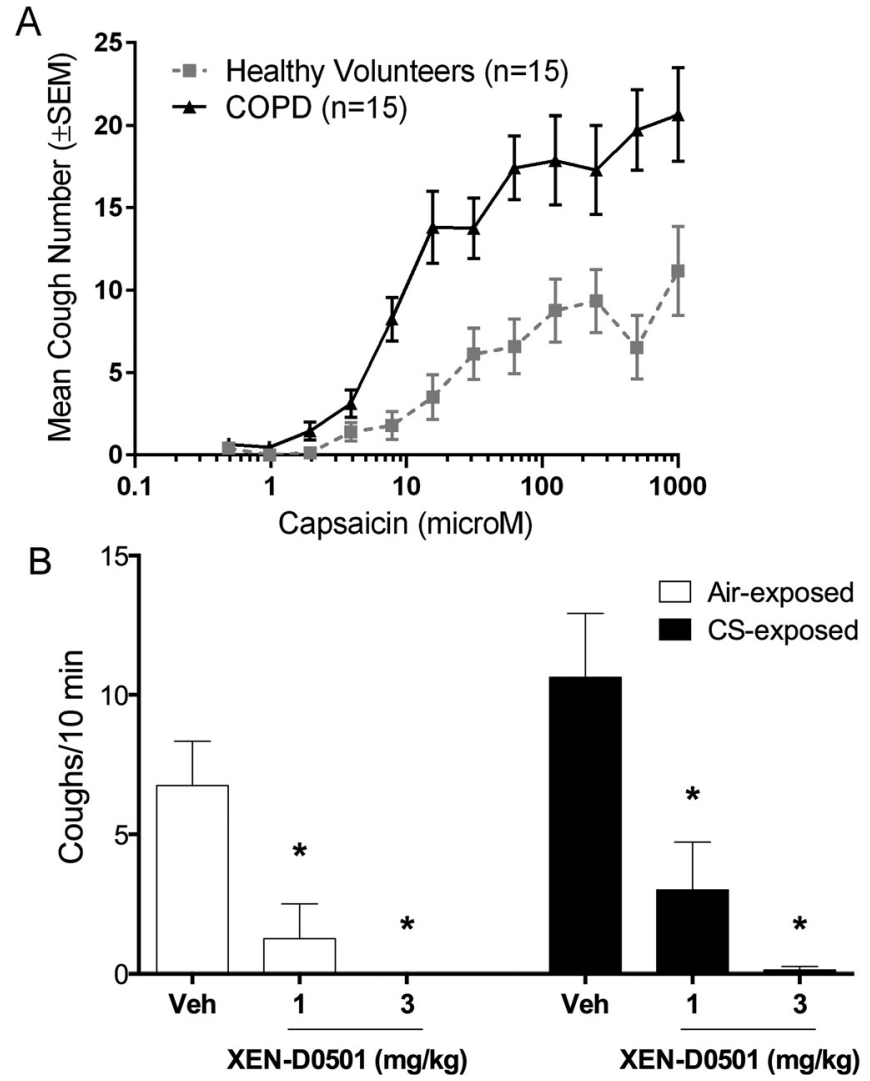

Abstract P4 Figure 1 (A) Human coughs to capsaicin - healthy controls; $n=15$, median age 56 (IQR 39-60) 5 females, mean FEV $109.5 \%( \pm 17.0)$. CDPD patient: $n=15$, median age $69(64-72), 5$ female, mean FEV $158 \%( \pm 11,2), p<0.001$ (General Estimating Equations. (B)Guinea-pig coughs to capsaicin $(30 \mu \mathrm{M}){ }^{*} \mathrm{p}<0.05$ compared to Veh control (Kruskal-Wallis with Dunn's post-test) $n=8$. 
Results Capsaicin-evoked cough was increased in COPD patients (Fig.1A) and in CS-exposed guinea-pigs (Fig.1B) compared to controls. Capsaicin induced greater depolarisation in nerve tissue from CS-exposed guinea-pigs, and in human vagus nerves from smokers, compared to controls. Capsaicin also induced greater $\left[\mathrm{Ca}^{2+}\right]_{\mathrm{i}}$ increases in airway-terminating jugular and nodose (which are normally capsaicin-unresponsive) neurons from CSexposed guinea-pigs. XEN-D0501 (i.p. $1 \mathrm{~h}$ before cough recording) almost completely inhibited the cough response to capsaicin in both air- and CS-exposed guinea-pigs (Fig.1B).

Conclusions CS-exposure evoked increased cough to capsaicin in guinea-pigs, mimicking the enhanced cough phenotype observed in COPD patients. This was paralleled by enhanced capsaicin responses in isolated vagus nerves and airway neurons from CS-exposed guinea-pigs and in human vagus from smokers suggesting the enhanced cough phenotype is due to increased TRPV1-mediated sensory nerve responsiveness. Inhibition of the CS-enhanced cough response by XEN-D0501 further implicated a role for TRPV1. This data, together with the finding that TRPV1 KO mice display less inflammation in a similar pre-clinical model of CS-exposure ${ }^{2}$, indicates the potential utility of TRPV1 antagonists in the treatment of COPD, which is currently being evaluated in an ongoing COPD clinical trial.

\section{REFERENCES}

1 Round. Br J Clin Pharmacol. 2011:72:921-931

2 Baxter ATS. 2014;A2079

\section{P5 LIPID-LADEN MACROPHAGES IN BRONCHOALVEOLAR LAVAGE FLUID ARE NOT DIAGNOSTIC OF AIRWAY REFLUX}

YA Hayman, L Sadofsky, S Faruqi, SP Hart, AH Morice. Department of Cardiovascular and Respiratory Studies, University of Hull and Hull York Medical School, Castle Hill Hospital, Cottingham, UK

\subsection{6/thoraxjnl-2014-206260.155}

Aims Demonstration of lipid-laden macrophages in respiratory secretions has been suggested to be a marker of reflux and aspiration. However studies looking at the diagnostic value of quantifying macrophage ingested lipids have been inconclusive. We wanted to look evaluate the utility of this technique in diagnosing airway reflux.

Methods In this prospective study bronchoalveolar lavage samples were collected from patient's undergoing flexible bronchoscopy for various indications (lung cancer, chronic cough, ILD etc). Cells collected were stained with Oil-Red-O. Lipid-laden macrophage index (LLMI) was used to quantify lipid accumulation. This is calculated by grading the amount of intracellular Oil-Red-O positive particles per 100 alveolar macrophages. A score of 0 (no opacification) to 4 (total opacification) is assigned to each macrophage. The sum of the scores yields the LLMI. Patients were asked to complete the Hull Airways Reflux Questionnaire (HARQ), a validated tool to diagnose airways reflux. One of the investigators, blind to the analysis, independently reviewed the clinical notes to establish a diagnosis of associated airway reflux. The investigator performing cell analysis was blind to the clinical details. The groups with and without a clinical diagnosis of airway reflux were compared. Correlations between the HARQ score and LLMI were sought.

Results Twenty nine patients (19 females, mean age 64 years) were included in the study. Of these in 11 a clinical diagnosis of associated airway reflux was made. The mean [SD] LLMI in the group with airway reflux (95[105]) was not significantly different from those without airway reflux (90[75]). There was a weak correlation observed between the HARQ score and the LLMI (0.09) which was not statistically significant $(\mathrm{p}=0.69)$.

Conclusions We fail to demonstrate significant association between LLMI and either a clinical diagnosis of airway reflux or the HARQ score. This could be due to the fact that macrophages scavenge both exogenous and endogenous material. However our study is limited by small numbers and disparate underlying clinical diagnoses. The small correlation of LLMI with HARQ scores merits further evaluation. Whether the proportion of macrophages phagocytising lipids or the degree of lipid ingestion by the macrophages is more important needs further study.

\section{P6 1 MENTHOL HAS BENEFICIAL EFFECTS IN THE AIRWAYS THROUGH A TRPM8-INDEPENDENT MECHANISM}

SA Maher, MA Birrell, SJ Bonvini, MA Wortley, ED Dubuis, F Shala, VC Jones, P Flajolet, Y Negreskul, Z Britton, L Hebib, MG Belvisi. Imperial College, London, UK

\subsection{6/thoraxjnl-2014-206260.156}

Introduction Asthma is a debilitating disease of the airways characterised by symptoms such as bronchoconstriction, hyperresponsiveness and cough. Current therapies are associated with significant side effects and are ineffective in severe disease highlighting the need for novel treatments. Menthol, a cooling compound found in medicinal products, is commonly thought to activate the thermo-sensing cation-permeable Transient Receptor Potential Melastatin 8 (TRPM8) channel. Furthermore, menthol is known for its beneficial effects in the airways such as bronchodilation $^{1}$ and suppression of nerve activation and cough, ${ }^{2}$ however the mechanism of action is unknown.

Aim To pharmacologically characterise the role of TRPM8 and menthol in the airways.

Methods TRPM8 gene expression was measured using Taqman real-time PCR. Mouse and guinea pig isolated vagal nerves were mounted in a grease-gap chamber and depolarisation $(\mathrm{mV})$ recorded as an indicator of sensory nerve activity. Segments of murine and guinea pig trachea were attached to a force transducer in an organ bath and relaxation of carbachol-induced tone recorded (mg).

Results TRPM8 is expressed in mouse and guinea pig vagal ganglia. The selective TRPM8 agonist, WS3, caused activation of guinea pig and mouse isolated vagal nerves, which was inhibited by the TRPM8 antagonist, JNJ41876666. Furthermore, WS3induced depolarisation was abolished in isolated vagal nerves from Trpm $8^{-/-}$mice. (-)-menthol (active form) caused a small depolarisation of mouse and guinea pig isolated vagal nerves, which was blocked by JNJ41876666. Interestingly, pre-incubation of (-)-menthol inhibited vagal nerve activation induced by the tussive stimulus, capsaicin, an effect that was not inhibited by JNJ41876666. WS3 and (-)-menthol caused a concentrationdependent relaxation of murine and guinea pig trachea, which was not abolished by JNJ41876666 nor in the Trpm $8^{-1-}$ mouse airway. No expression of TRPM8 was detected in guinea pig or mouse airway smooth muscle.

Conclusions (-)-menthol caused a small TRPM8-dependent activation but a robust TRPM8-independent inhibition of vagal sensory nerve activity and relaxation of airway smooth muscle. Elucidating the mechanism behind the beneficial effects of (-)menthol could lead to the development of promising new therapeutic targets for airway diseases such as asthma. 